\title{
Laboreal
}

Volume $16 \mathrm{~N}^{\circ} 1 \mid 2020$

Quando o trabalho real é tabu

\section{Jacques Leplat : la psicología del trabajo en el} \section{corazón}

Jacques Leplat : a psicologia do trabalho no coração

Jacques Leplat : la psychologie du travail au cour

Jacques Leplat : the work psychology in the heart

\section{Annie Weill-Fassina}

Traductor. Gabriela Cuenca

\section{OpenEdition}

\section{Journals}

\section{Edición electrónica}

URL: http://journals.openedition.org/laboreal/16177

DOI: 10.4000/laboreal. 16177

ISSN: 1646-5237

Editor

Universidade do Porto

\section{Referencia electrónica}

Annie Weill-Fassina, « Jacques Leplat : la psicología del trabajo en el corazón », Laboreal [En línea]

Volume $16 \mathrm{~N}^{0} 1$ | 2020, Publicado el 01 julio 2020, consultado el 08 julio 2020. URL : http:// journals.openedition.org/laboreal/16177 ; DOI : https://doi.org/10.4000/laboreal.16177

Este documento fue generado automáticamente el 8 julio 2020.

\section{(c) (7) (9)}

Laboreal está licenciado com uma Licença Creative Commons - Atribuição-NãoComercial 4.0 Internacional. 


\section{Jacques Leplat : la psicología del trabajo en el corazón}

Jacques Leplat : a psicologia do trabalho no coração

Jacques Leplat : la psychologie du travail au cour

Jacques Leplat : the work psychology in the heart

\section{Annie Weill-Fassina}

Tradución : Gabriela Cuenca

\section{NOTA DEL EDITOR}

Traducción : Gabriela Cuenca - gcuenca@frba.utn.edu.ar

\section{2-1980 : El tiempo de una trayectoria profesional}

1 Casi 30 años han transcurrido entre el artículo de 1952, titulado "La adaptación de la máquina al hombre" publicado anteriormente en Laboreal y el artículo "La psychologie du travail : un aperçu" publicado en 1980 en un número especial del Bulletin de Psychologie dedicado a la psicología laboral. Mientras tanto, Jacques Leplat colaboró con Jean-Marie Faverge (1912-1988) en el CERP (Centre d'Études et de Recherches en Psychotechnique). Lo sucedió como director en 1958, después de su partida a la Universidad de Bruselas tras la muerte de André Ombredane. (1898-1958). Este departamento rebautizado "Centre d'Études et de Recherches Psychologiques" deja entonces de orientar y seleccionar becarios formados en ANIFRMO [ ${ }^{1}$, pues será función de otro departamento. Su nueva misión es evaluar las técnicas de formación de esta organización y asesorar empresas. Paul Albou, en una entrevista que concederá más tarde a la Comisión de Historia de la SELF [ $\left.{ }^{2}\right]$, dirá : "Para la Comisión de la Productividad, organismo del Ministerio de Trabajo, el CERP era un motor para las investigaciones y reflexiones sobre el trabajo... Era necesario estudiar las 
condiciones físicas, materiales o de otro tipo en que se realizaba el trabajo así como las relaciones humanas en la empresa. Se trataba de adaptar el trabajo al hombre" (Albou, 2000).

2 En 1966, Jacques Leplat deja el CERP, reemplaza a Raymond Bonnardel (1901-1988) en la École Pratique des Hautes Études (EPHE) y dirige el "Laboratoire de Psychologie Appliquée" ubicado en el 41 de la rue Gay Lussac, en el Institut national d'étude du travail et d'orientation professionnelle (INETOP). Lo rebautiza como "Laboratoire de Psychologie du Travail", modificando su campo y su propósito. En este contexto y desde el inicio, Leplat explicita que los trabajos del Laboratorio no estarán "orientados esencialmente hacia la solución de problemas prácticos (pues llevaría a una concepción errónea y acotada de la psicología del trabajo)" (pág. 197) sino hacia la investigación básica y la enseñanza a través de la investigación centrada en los aspectos psicológicos de la "actividad" o de la "conducta" de trabajo. Leplat amplia en el artículo presentado, esta diferencia entre investigación e intervención siendo uno de los puntos estructurantes de sus orientaciones. El Laboratorio forma parte del movimiento de reorientación de la psicología del trabajo remarcado por M. Reuchlin (1971) en el Traité de Psychologie appliquée, en un capítulo al cual Leplat refiere en su artículo de 1980 (p.199): "La importancia de los estudios diferenciales basados en pruebas que constituían el grueso de los antiguos libros de texto se ha reducido mientras que el papel de la psicología ergonómica y la psicología de las organizaciones ha aumentado considerablemente".

3 En 1980, encontrándose a 9 años de iniciar su estudiosa jubilación, que dedicó hasta hace poco a escribir resúmenes y reeditar trabajos que considera como los más significativos de este período de la historia de la psicología del trabajo y de la cual siempre ha querido construir un estatus científico en áreas de su interés como el análisis del trabajo, la seguridad y la formación (Leplat, 1980, 1992, 1993, 1997, 2008). Ya en 1980, este número especial -del Bulletin de Psychologie- aún disponible (344) - reúne numerosos artículos escritos por investigadores y profesionales diseminados por todo el territorio nacional, que testimonian la existencia de una red cercana al Laboratorio y la vitalidad de la psicología laboral en Francia. Por eso, en lugar de analizarlo, prefiero mencionar algunas de las principales líneas de investigación que han sido desarrolladas en los equipos dirigidos por Jacques Leplat.

\section{En los años 60, en el CERP, preguntas derivadas de los cambios del trabajo}

4 En este período, desde un punto de vista sociológico, las transformaciones del mundo industrial afectan particularmente ciertas poblaciones. En el CERP, algunos equipos administrativamente vinculados a Jacques Leplat se hacen eco. Así, Guy Barbichon (1968) entre otros, realiza encuestas sobre el futuro de los campesinos fuera de la agricultura y sobre el movimiento de la población rural hacia el mundo industrial. Françoise Lantier (1970) investiga las dificultades de las mujeres al acceso del mercado laboral y a los empleos calificados. Jean Grisez (1980) estudia el empleo y el futuro de los técnicos formados en la Institución.

Desde un punto de vista sociotécnico, las transformaciones del trabajo vinculadas a la automatización y la informatización implicaban cada vez más información, signos y símbolos. Como lo explicará más tarde Leplat (1997, p. 100), "Los psicólogos están interesados en los nuevos tipos de tareas cuyo diseño y ejecución plantean problemas a los 
ingenieros y organizadores. Estas nuevas tareas en particular están determinadas por el hecho de que los operadores no intervienen directamente sobre los objetos a transformar, sino que lo hacen a través de dispositivos que comunican tanto la información procedente del campo de trabajo como las acciones llevadas a cabo en él. Estas tareas requieren una actividad cognitiva cada vez más grande cuyo análisis se vuelve cada vez más necesario".

Son características de este cambio las investigaciones iniciadas por el equipo de investigación vinculado directamente a LepLat. Los pedidos de intervención muestran que los análisis del comportamiento en el marco conductista- predominante hasta ese momento - ya no eran pertinentes para explicar lo que sucedía en terreno. Por esta razón y en el marco de un proyecto de automatización de la navegación aérea, para diseñar una ayuda para la gestión de las rutas aéreas para los "controladores de tráfico aéreo" fue esencial comprender la información que necesitaban, bajo qué forma, sus modos de razonamiento, en síntesis, actualizar una actividad no observable cuyo requisito principal fuera la seguridad de los aviones (Bisseret y Leplat 1965 ; Bisseret, 1995). En las acerías, en los diversos sistemas de trabajo, como el transporte o el monitoreo a distancia, los análisis centrados en la seguridad y la prevención de accidentes ya no implicaban problemas de vigilancia o de predisposición, sino problemas de comunicación dentro o entre equipos (Cuny, 1967). Otras investigaciones cuestionaron el diseño, la comprensión y el uso de las "ayudas de trabajo" (tablas, interfaces gráficas, guía de acción,...) que plantearon problemas de comprensión y de uso en situaciones de trabajo y de entrenamiento. (Weill-Fassina, 1968).

El punto en común de estos problemas era orientar la investigación hacia la psicología cognitiva enfocándose en las actividades mentales, en la toma y procesamiento de la información, en la representación de situaciones, en los procesos de toma de decisiones en distintas situaciones laborales. La interpretación teórica de las observaciones se desarrolló en tres direcciones diferentes que según los investigadores parecían las más relevantes para abordar el problema planteado: la decisión y la resolución de problemas de Newell y Simons, la semiología y el desarrollo de las representaciones del espacio en la línea con los trabajos de Piaget. Este movimiento de la observación empírica hacia las teorías caracterizó este primer período que podría describirse como ergonomía psicológica.

\section{En los años 70, en el Laboratorio de Psicología del trabajo, la importancia de los marcos teóricos}

El equipo del CERP ha sido disuelto. El laboratorio de psicología del trabajo de la EPHE se convierte en un equipo de investigación asociado (ERA) en el CNRS bajo el nombre de "Centro de estudios psicológicos del hombre en su ambiente sociotécnico". Los investigadores que participan en este nuevo equipo pertenecen a esta organización conocida por estar orientada a la investigación fundamental. Como señala Leplat en el artículo de 1980 (p.199) : "En los últimos años, por otro lado, hubo una creciente preocupación por vincular los estudios con los marcos teóricos, algunos de los cuales son comunes a los de la psicología y otros, más específicos al campo del trabajo. Este movimiento, que aún no ha alcanzado toda su magnitud, se ve alentado por el interés de los psicólogos, más orientados hacia aspectos teóricos generales que a abordar los problemas laborales". 
Conceptos tales como la brecha entre la tarea prescrita y la tarea real o entre la tarea y la actividad, el error, la representación, la regulación del sistema, la regulación de la actividad, la fiabilidad humana, la fiabilidad del sistema, la anticipación, la planificación, se encuentran en el centro de debates y controversias en referencia a los diversos marcos teóricos elegidos por los investigadores : La teoría de la acción de Leontiev (Pailhous, 1970; Savoyant 1985), los sistemas de representación y procesamiento de la información (Hoc, 1972); las tentativas de aplicar la teoría de Piaget sobre el desarrollo del funcionamiento cognitivo del niño al adulto enfrentado a nuevas situaciones (Vermersch, 1978; Weill-Fassina, 1988). Al mismo tiempo, se han abierto nuevos campos de estudio que aluden a la multicausalidad de los accidentes (Leplat y Cuny, 1974), al trabajo en equipo colectivo (Savoyant, 1984) y a los efectos sociales y de salud de los horarios atípicos de trabajo (Gadbois y Queinnec, 1984) e invitan a ampliar las observaciones más allá del puesto aislado y a interrogarse sobre la organización del trabajo.

\section{Una revisión programática de la psicología del trabajo}

Esta dinámica de investigación llevada a cabo en los equipos del CERP y en el Laboratorio de Psicología Laboral (esquematizada rápidamente aquí y sin pretender ser exhaustiva) y las convergencias de los estudios publicados en este mismo número del boletín de psicología (344), están implícitos en el artículo escrito por Leplat en 1980 e incluidos en este número de Laboreal. Sus reflexiones sobre la psicología del trabajo representan una revisión y un manifiesto. En el cuadro 1 se delinean, categorizan y estructuran los campos de trabajo que probablemente interpelen diferentes campos de la psicología del trabajo. Además de la diferencia entre la psicología del trabajo y la intervención ya mencionada, surgen dos aspectos fundamentales :

- La actividad laboral siempre se considera en interacción con los instrumentos y el entorno técnico inmediato y/o las condiciones (en lugar de "determinantes") organizacionales o sociales [3]. Fiel a la separación tradicional entre psicología experimental y psicología clínica (Fraisse, 1956), Leplat siempre se focalizó en el análisis cognitivo de los procesos mentales involucrados en el trabajo. Aspectos subjetivos de la conducta de las emociones encontraron luego un lugar en sus preocupaciones (Clot y Leplat, 2005).

- El análisis objetivo de los comportamientos y los métodos de tipo experimental son elegidos para determinar el papel de las condiciones de trabajo tomadas en cuenta en la conducta, como garantía de un método científico. Lo que ha llevado frecuentemente a Jacques Leplat a responder, como en este artículo, al problema del reduccionismo que plantea la transición de las observaciones de terreno a la experimentación. Esto ha sido (y sigue siendo) objeto de múltiples controversias entre los psicólogos generalistas, los defensores del análisis ergonómico del trabajo y los partidarios de un análisis que tenga en cuenta la subjetividad de los participantes.

11 Ampliando estas discusiones, en su último libro, Mélanges d'ergonomie: activité, compétence, erreur (2011) con un título evocador, Leplat retoma y comenta los últimos textos que han sido relevantes para él. Siguiendo a Pierre Rabardel (2005), vuelve (p. 60-61) sobre la diferencia entre capacidad y poder para actuar. Para él, "el poder de actuar es también transformar las condiciones internas y externas para llevar a cabo la tarea. No se trata de una tarea definida por única vez, sino de una tarea que el sujeto redefine de acuerdo con los propósitos y los medios que se da a sí mismo (o que le dan). 
Por lo tanto, plantea la cuestión del desarrollo de los individuos y de los lugares de trabajo, que ha sido un denominador común de muchas investigaciones durante la última década. También se posiciona sobre un tema que ha suscitado una serie de debates y trabajos en el laboratorio de ergonomía (Falzon, 2005) y en el equipo de Psicología del trabajo y clínica de la actividad (Clot, 2008) del Centre d'Études sur le travail et le développement (CRTD) del Centre National des Arts et Métiers y que sigue siendo objeto de debates psicológicos, sociales, políticos y filosóficos.

Dejemos que Jacques Leplat concluya esta nota citando la respuesta dada a Antoine Laville, -sucesor del Laboratorio, ahora "laboratorio de ergonomía fisiológica y cognitiva "-, en la entrevista que concedió en 2000 a la Comisión de Historia del SELF : "Un problema práctico siempre es multidisciplinario, tiene múltiples dimensiones y podemos analizarlo de varias maneras. No hay ninguna teoría o modelo que por sí solo agote la complejidad de un caso. Un problema práctico, un problema de campo no es sólo un modelo, tiene una riqueza que no puede reducirse a una disciplina o a un único punto de vista. Hay una actitud de apertura que es crucial... Mi punto de vista es que un modelo es esencial en un estudio, en una investigación, pero que siempre será una visión parcial de la realidad que necesariamente la desborda. Es bueno tener uno o más modelos preferidos, pero significa ver los problemas con un determinado par de anteojos y no debemos creer que es el único, debemos estar siempre atentos a lo que queda sin explicación ».

\section{BIBLIOGRAFÍA}

Albou, P. (2000). Entrevista com Antoine Laville. Commission histoire de la SELF. https:// ergonomie-self.org/wp-content/uploads/2016/09/Paul-ALBOU.pdf.

Bisseret, A. (1995). Représentation et décision experte. Psychologie cognitive de la décision chez les aiguilleurs du ciel. Toulouse, Octarès-Éditions.

Barbichon, G. (1968). Aspects psychologiques et sociaux du transfert des agriculteurs de l'agriculture vers l'industrie. Économie rurale,77, 1, 45-54.

Clot, Y. (2008). Travail et pouvoir d'agir. Paris : PUF.

Clot, Y. \& Leplat J. (2005). La méthode clinique en ergonomie et en psychologie du travail. Le travail humain, 68, 4, 289- 316.

Cuny, X. (1967). La circulation de l'information dans un système élémentaire d'un service de transport. Bulletin du CERP, 16, 1-19.

Cuny, X. (1969). Sémiologie et études ergonomiques des communications de travail. Le Travail humain, 32, (3-4), 177-198.

Falzon, P. (2005). Ergonomics, Knowledge Development and the Design of Enabling Environments. Proceedings of the Humanizing Work and Work Environment. HWWE', 2005, Conference. Guwahati, India, 10-12 décembre, 1-8.

Fraisse P. (1956). Defensa do método experimental em Psicologia. Em P. Fraisse, Manuel pratique de psychologie expérimentale [p. 1-42]. Paris : PUF. 
Hoc, J.M. (1972). Représentation mentale et modèles cognitifs de traitement de l'information. Le Travail humain, 35-1, 17-36.

Gadbois C. \& Queinnec Y. (1984). Travail de nuit, rythmes circadiens et régulation des activités. Le Travail Humain, 47, 3, 195-225.

Grisez, J. (1980). Emploi et comportement de mobilité. Bulletin de psychologie, 33, 344, 4, 11, 417- 420.

Lantier, F. (1970). Les attitudes sociales et industrielles concernant l'accès des femmes aux emplois qualifiés et leur avancement professionnel: Incidences du développement technologique. Paris. Association Nationale pour la formation des adultes. Centre d'Études et de Recherches Psychotechniques (65p.).

Leplat J. (1980). La psychologie ergonomique. Paris : PUF.

Leplat, J. (1992/1993). L 'analyse du travail en psychologie ergonomique (T. 1 et 2). Toulouse : Octarès Editions.

Leplat, J. (1997). Regards sur l'activité en situation de travail. Contribution à la psychologie ergonomique. Paris : PUF. Coll. le travail humain.

Leplat, J. (1997). L'analyse psychologique du travail : Quelques jalons historiques. Dans J. Leplat, Regards sur l'activité en situation de travail. Contribution à la psychologie ergonomique (p. 91-108). Paris : PUF. Coll. le travail humain. [Artigo publicado em Le Travail humain, 1993, 2-3, 115-131].

Leplat, J. (2000). Entrevista com Antoine Laville. https://ergonomie-self.org/vie-de-la-self/ actualite-des-commissions

Leplat, J. (2008). Repères pour l'analyse de l'activité en ergonomie. Paris : PUF. Collection travail humain.

Leplat, J. (2011). Mélanges d'ergonomie : activité, compétence, erreur. Toulouse : Octarès Editions.

Leplat, J. \& Bisseret, A. (1965). Analyse des processus de traitement de l'information chez les contrôleurs de la navigation aérienne. Bulletin du CERP, 14, 51- 68. [Reproduzido em J. Leplat, 1995, L'analyse du travail en psychologie ergonomique [p. 215-216]. Tome 2. Paris : PUF].

Leplat, J. \& Cuny, X. (1974). Les accidents du travail. Paris : PUF.

Pailhous. J. (1970). La représentation de l'espace urbain. Paris : PUF.

Rabardel, P. (2005). Instrument subjectif et développement du pouvoir d'agir. Em P. Rabardel e P. Pastré (dir.), Modèles du sujet pour la conception (p. 11-30). Toulouse : Octarès Editions.

Reuchlin, M. (1971). Traité de psychologie appliquée. Tome 1. Les applications de la psychologie. Paris : PUF.

Savoyant, A. (1984). Définition et voies d'analyse de l'activité collective des équipes de travail. Cahiers de psychologie cognitive, 4,273-284.

Vermersch, P. (1978). Une problématique théorique en psychologie du travail. Essais d'application de la théorie opératoire de l'intelligence de J. Piaget à l'analyse du fonctionnement cognitif. Travail Humain, 2,41, 265-278.

Weill-Fassina, A. (1968). Un intermédiaire dans le système homme-travail, lecture et écriture de schémas explicatifs. Soutenance de thèse. Bulletin de Psychologie, XXIII, 17,286,1129-1132.

Weill-Fassina, A. (1988). Complexité figurale et complexité opératoire dans la compréhension et l'utilisation de graphismes techniques. Bulletin de psychologie, XLI, 386,645 -653. 


\section{NOTAS}

1. Association nationale interprofessionnelle de formation et de reconversion de la main d'œuvre. Actual AFPA - Asociación para la formación de Adultos.

2. Sociétè d'Ergonomie de Langue Française.

3. El término "determinante" utilizado tradicionalmente implica considerar que existe una fuerte causalidad que no permite a los protagonistas modular su actividad.

\section{AUTORES}

\section{ANNIE WEILL-FASSINA}

Groupe de Recherches sur l'Histoire du Travail et de l'Orientation, Centre d'Études sur le travail et le développement, Centre National des Arts et Métiers, 41 Rue Gay-Lussac, 75020 Paris weill.fassina@orange.fr 